\title{
Nursing opinion leadership: a preliminary model derived from philosophic theories of rational belief
}

\author{
Christine A. Anderson* $\mathrm{PhD}$ and Ann L. Whall ${ }^{\dagger} \mathrm{PhD}$
}

*Clinical Assistant Professor, School of Nursing, University of Michigan, Ann Arbor, and †'Maggie Allesee Endowed Professor in Gerontology, School of Nursing, Oakland University, Rochester, MI, USA others' decisions about adopting new products, practices or ideas. In the healthcare setting, the importance of translating new research evidence into practice has led to interest in understanding how opinion leaders could be used to speed this process. Despite continued interest, gaps in understanding opinion leadership remain. Agent-based models are computer models that have proven to be useful for representing dynamic and contextual phenomena such as opinion leadership. The purpose of this paper is to describe the work conducted in preparation for the development of an agent-based model of nursing opinion leadership. The aim of this phase of the model development project was to clarify basic assumptions about opinions, the individual attributes of opinion leaders and characteristics of the context in which they are effective. The process used to clarify these assumptions was the construction of a preliminary nursing opinion leader model, derived from philosophical theories about belief formation.

Keywords: epistemology, philosophy of science, nursing theory.

\section{Introduction}

Today's focus on evidenced-based practice in the provision of nursing care requires that nurses be current on various types of information and able to enhance the application of information into practical use. According to the Institute of Medicine in Crossing the Quality Chasm (Institute of Medicine Committee on

Correspondence: Dr Christine A. Anderson, Clinical Assistant Professor, School of Nursing, University of Michigan, $400 \mathrm{~N}$. Ingalls, Ste. 4170, Ann Arbor, MI 48109, USA. Tel.: +1 734615 0530; fax: +1 734647 2416; e-mail: fauve@umich.edu
Quality of Health Care in America, 2001), the average time it takes for the results of randomized controlled trials (RCT) to reach practice application is 17 years. Enhanced dissemination is needed. Rogers (2003) in his influential work about the Diffusion of Innovations posits that identifying and using opinion leaders, those individuals who lead in influencing others' opinions, may help speed adoption of new practices.

The literature concerning opinion leadership (OL) is vast, encompassing many decades of research on the topic by investigators in many diverse fields (Greenhalgh et al., 2005). Despite continued interest, gaps remain in understanding the phenomenon and a 
number of methodological issues have been identified. Within health care, a Cochrane review (Doumit et al., 2007) of 12 RCT using OL as an intervention strategy to change practice behaviours found that the effectiveness of OL is mixed since some of the studies did not show a significant outcome. Of the 12 studies, only one was identified as being at low risk for bias, while eight were considered high risk based on methodological issues such as the randomization process and outcome assessment. Other important limitations included a lack of clear definition of the role of the OL resulting in problems for replication and inconsistencies that make it difficult to understand the attributes of an effective OL. In addition, there is the potential for OL to change over time. Doumit et al. (2007) identified several implications for future research. The need to identify the context in which OL are more effective, studies to assess the methods of identification of OL and clarifications of the actual activities used by OL are examples (Doumit et al., 2007).

Greenhalgh et al. (2005) reviewed qualitative and mixed-method studies as well as RCT and concluded that OL was important to implementation projects. The characterization of an OL as emergent and informal with a lack of distinctive role boundaries was a key finding. The authors advocated using multiple methods to provide a more complete picture of a complex, multifaceted phenomenon that is interactive with many factors, including the context (Greenhalgh et al., 2005).

Agent-based modelling is a theory development tool that is useful for representing dynamic, multilevel and contextual phenomena such as OL (Anderson \& Whall, 2011). Agent-based models are computer programmes that allow representation of individual 'agents' and the processes used by them in a given environment. Computer modelling of this type requires precision in terms of defining agent characteristics and logical consistency of agent behaviours in order for the model programme to actually work (Miller \& Page, 2007; Gilbert, 2008). The purpose of this paper is to describe the work conducted in preparation for the development of an agent-based model of nursing OL. The agent-based model is designed to generate the phenomena of OL. Dynamic simulations, whereby various individual and contextual variables are manipulated, are used to explain which factors (or combination of factors) contribute to the emergence of an individual opinion leader over time. The aim of this phase of the model development project was to clarify basic assumptions about opinions, the individual attributes of potential opinion leaders and characteristics of the context in which they may be effective. The process used to clarify these assumptions was the construction of a preliminary nursing opinion leader model, derived from philosophical theories about belief formation.

\section{Preliminary model development methods}

The basic framework for this phase of the model development effort is a combination of the theory derivation (TD) and theory synthesis (TS) techniques described by Walker \& Avant (1983, 1988, 1995, 2005). Although as Risjord (2009) explains, Walker and Avant's view of concepts in theory construction is believed by some to be problematic because it does not adequately address the effect of context on conceptual meaning, their methods are well known to the nursing community. Since our plan involved the use of agent-based modelling, a new method used in other fields to address contextual issues, we adapted Walker and Avant's familiar and straightforward techniques for use as a starting point for our effort. According to Walker \& Avant (2005), derivation is the process of using analogies to explain or predict; it is useful for developing new theoretical insights about phenomena. In the case of OL, the aim is to develop a better understanding of the processes used by opinion leaders to revise their own beliefs and to influence the opinion of others. The intention of beginning the agent-based OL model development with a clear specification of assumptions leads to the use of this approach. The steps for TD described here are: (1) the selection of source theories for derivation; (2) the identification of useful structure and content from the source theory; and (3) modification of the content and structure, for use in nursing (Walker \& Avant, 2005). The use of Walker \& Avant's (2005) synthesis method included a comparison of the relational 
statements from the selected theories for conceptual similarities. Next, organization of these statements in terms of antecedents and effects was developed. Finally, the construction of a representation in the form of a model diagram suitable for guiding computer programming revealed the integration of the statements from both theories (Walker \& Avant, 2005).

\section{Selection of source theories}

Because opinion leaders are individuals whose degree of influence in changing the opinion of others may be related to their social context, theory selection began with examination of the philosophical roots of opinion formation by both individuals and groups. Bayesianism is a philosophic theory concerning the rational beliefs of individuals (Joyce, 2004). Kitcher's (1993) theory of the Organization of Cognitive Labor (OCL) is concerned with the ways in which groups of people arrive at consensus practices via individual group members' decisions about those practices. Kitcher's theory was selected because it is an effort to explain the role of the social context on the individual (as well as the effect of the individual on the community), in the adoption of new research findings.

Both theories, Bayesianism and the Organization of Cognitive Labor (B \& OCL), are normative (e.g. are not the result of empirical data), as well as abstract in nature. The development of each theory relied on the extensive use of logical argumentation and mathematical formalism as philosophic methods to explain and justify conceptual relationships. The abstract nature and formal logical justifications of B \& OCL contributed to their selection as source theories since they offered a different approach for theory development (e.g. normative instead of empirical).

The next step in Walker \& Avant's (2005) TD method is to identify the useful content and structure in the source theory. To do so, an examination of the meanings of B \& OCL, as described by Joyce and Kitcher, proceeded through the identification of their concepts and relationship statements (Hardy, 1973/ 2004). Following this analysis, synthesis of portions of the two theories was completed.

\section{Identification of useful structure and content}

The analytical process of identifying the concepts, their meanings and relationships, within B \& OCL, began with listing and defining major ideas and their definitions. The process of translating mathematical formalism into words in order to create brief summaries of the content and structure of B \& OCL resulted in the simplification and prioritization of the concepts and the relationships between them. The summaries below are followed by a discussion of the results of the analysis and synthesis.

\section{Bayesianism}

According to Joyce (2004), Bayesianism is a normative theory of rational belief. Rational beliefs are variable in strength, consistent with the laws of probability, and can change through the process of learning (Joyce, 2004). From an epistemic standpoint, rational agents seek to maximize the accuracy of their beliefs. Practically motivated rational agents want to maximize their subjective expected utility, or determine what is in their best interest. The focus of this synopsis is on Bayesian epistemology as described by Joyce $(1998,2004,2005)$.

Joyce (2004) defines a belief as an individual's estimated level of confidence in the truth value of a proposition, expressed in probabilistic terms. Belief corresponds to the extent to which a person is willing to presuppose the truth of a proposition in theoretical or practical reasoning. It is not necessary to assign a specific numerical value to express the strength of a belief probabilistically (e.g. Jane believes it is improbable that $\mathrm{X}$ is true). Beliefs can be unconditional or conditional. An unconditional belief means that confidence is based a particular proposition, whereas a conditional belief is the expression of confidence in the truth of one proposition while supposing that other propositions are also true (e.g. $\mathrm{X}$ is highly probable given Y) (Joyce, 2004).

The quality of a person's belief is evaluated by the accuracy of his subjective confidence estimates relative to objective probabilities. Individuals who have high levels of confidence in the truth are rewarded because using true premises (accurate representation of the world) as a basis for reasoning, in theory, 
produces better outcomes (Joyce, 2004). Individuals are motivated to seek accurate beliefs, and increased accuracy follows from holding beliefs that are consistent with the laws of probability (Joyce, 1998).

Joyce (2005) further describes an individual's belief as a reflection of that person's total evidence in favour of a given proposition. Total evidence is relative to the individual because it includes both prior beliefs and new knowledge gained through learning. Bayes's theorem is a rule for calculating the probability of a person's revised belief (termed 'posterior' belief) while taking into account new evidence. Bayes's theorem relates this posterior probability of a hypothesis, which is conditional on the new information, to the ratio of the 'prior' probability (e.g. prior to the new evidence) of the hypothesis and the probability of the new information. This ratio is then multiplied by the 'likelihood' of the new information given what is already known about the hypothesis (Joyce, 2004).

Joyce (2005) claims that three attributes of evidence affect beliefs in different ways. The first is balance, an assessment of the truth value of the evidence, which is either in favour of or opposed to the belief. Balance corresponds to the strength of the belief. Second, the weight or amount of evidence affects the stability of a belief, e.g. the greater the weight of the accumulated evidence, the less likely a dramatic change in belief will occur. Finally, specificity refers to the degree to which the evidence is complete or unambiguous with respect to the proposition. Specificity influences the range of potential probabilistic values of the person's state of confidence in the belief. Very specific evidence may result in a precise degree of change, whereas ambiguous evidence results in a broad range of plausible new confidence probabilities (Joyce, 2005).

Balance, weight and specificity of evidence play a role in learning, or conditioning. Conditioning is the process of adjusting a belief based on the acquisition of new evidence while taking into account the degree of effect of the prior belief in the determination of the new level of confidence. The learning process takes place in two stages. First, the new information causes a subset of beliefs to be altered. Following this experience and accounting for what he or she knows, the person will revise other opinions.
Simple conditioning is the basic model of Bayesian leaning. In this case, a person with a prior belief about a proposition $\mathrm{X}$ that has a probability $>0$ and $<1$ (uncertainty) undergoes a learning experience where the new information causes the person to be certain ( $100 \%$ probability) of X. It then follows that opinions inconsistent with $\mathrm{X}$ will be revised to probability $=0$, and opinions consistent with $\mathrm{X}$ will increase in probability. Simple conditioning requires certainty, in order to adjust beliefs when the evidence is vague or imprecise (not categorical); realistic learning involves gradated estimates of confidence in (beliefs about) the new evidence as well as the gradated original belief (Joyce, 2004).

\section{Kitcher's theory of the organization of cognitive labour}

Whereas Bayesian epistemology focuses on the individual, Kitcher's (1993) conception of belief revision includes the effects of the social interactions of individuals on the generation of consensus practices or community-wide beliefs (Kitcher, 1993). Social epistemology, according to Kitcher, is concerned with identifying the characteristics of social systems that are conducive to achieving collectively true beliefs. Communities also have practical aims, e.g. the optimal use of resources to enable best practices.

Kitcher (1993) describes the theory of OCL in terms of a community of scientists, and the ways in which the actions of individuals, in relation to the group, result in the achievement of community goals. The best community response to new scientific findings is the result of various distributions of individual effort or 'cognitive labour' relative to the new evidence.

Since there is no centralized controller to assign the work, Kitcher (1993) further explains how various combinations of individual decisions and social interactions are more or less successful in the generation of an ideal distribution of effort for attaining community goals. Central issues include the effect of motives, trust, authority and diversity on belief revision. Within the context of the social structure of the community, belief revision results from the cognitive work of scientists who when presented with new evidence can adopt it, ignore it or attempt to replicate it. The 
numbers of individuals in a community who align themselves with each of these options define the distribution of cognitive effort. Diversity means that the community is composed of individuals aligned with the various options concerning the new evidence.

Kitcher (1993) begins his discussion of OCL with an identification of the effects of authority on the knowledge development of individuals. These epistemic effects include depending on the teaching of authority figures, trusting others to decide certain issues and assessing the authority of others when making decisions regarding acceptance of the claims made by them. Among the advantages of deferring to authority are increasing the speed and feasibility of reaching goals. Borrowing, or deriving material from experts, for example can save the individual the time and effort required to obtain the information himself. Kitcher further posits that the decision to rely on authority includes an assessment of the expertise of the authority figure relative to the individual him/ herself, the resources available and the potential for obtaining credit by competing.

According to Kitcher (1993), the evaluation of an individual's authority by others rests on the assessment of the probability that what the individual says is true, or his/her credibility. The bases of an individual's credibility are the unearned authority resulting from social position, earned authority based on performance and personal authority based on relationships. The evaluator subjectively weights the importance of each type of authority and combines them to arrive at the total authority. Kitcher claims that the differentiation between authority and credit is important because there are three sources of authority whereas the attribution of credit results from an overall assessment of the variously weighted authority types. Seeking credit can be an important practical motivator for individuals engaged in scientific research.

The connection between credit and authority is particularly evident in the case where positive performance (earned authority) results are available to others who are making their assessments. Because earned authority is topic-relative (e.g. subject matter expertise), comparing the truth value of an authority's opinion to one's own beliefs on the topic is an impor- tant source of credit attribution. According to Kitcher (1993) when an individual evaluator is able to directly calibrate opinions in this way, due to his/her own knowledge about the topic, it leads to a baseline decision rule that favours following the judgement of the person with higher earned authority.

Unearned authority, on the other hand, is based on factors such as title or institutional affiliation, and can result in what Kitcher labels a 'prestige effect'. Personal authority is sometimes considered as a dimension of unearned authority because it is based on factors such as friendship ties. The weight assigned by an individual to unearned versus earned authority is a subjective assessment influenced by the importance the individual attaches to prestige, and the opportunity available to the person for direct calibration of earned authority.

Kitcher (1993) posits that it is often the case that direct calibration may be unreliable, e.g. someone outside the specialty or otherwise unknown to the person making the judgement introduces the new information. In these instances, indirect calibration may take place. Indirect calibration involves using the judgement of others, whom the individual believes to be knowledgeable about the source, when assessing authority.

To summarize Kitcher's major points, when a scientist is presented with new evidence, decisions about a course of action (adopt, ignore, further research) are based on epistemic goals for truth (maximize being right) and/or non-epistemic goals that maximize utility (e.g. be the first to be right). Secondly, the 'authority effect' means that an individual's decisions involve an assessment of his/her own credibility (which includes factoring in the perception of others), the credibility of his information sources and the credibility of his competitors. Thirdly, Kitcher posits that competition promotes diversity in a community. This is because some individuals will opt to adopt new findings, while some will determine that it is beneficial to create their own findings. Authority effects can reduce competition and therefore decrease diversity (Kitcher, 1993).

Next, Kitcher addresses the question of how diversity affects the attainment of community goals using examples describing the community response to 
innovation and the selection among rival experimental methods. In each example, the proposed optimal strategy for achieving community goals is followed by a discussion about how individual actions contribute to achieving the community goal.

The first example is that when there is an announcement of an innovative finding in a community, the best strategy for the community is the one that results in using the findings if true, rejecting them if false or delaying a decision pending further information. The extent to which consensus strategy is best depends on the probability that the finding is true. In most cases, some effort at replication is desirable.

The cost/benefit assessment related to using the resources to replicate the findings depends on the probability the finding is true, the reliability of potential replicators and the resources available. The chance that the optimal community response will arise, via individual community member action, depends on structural factors that contribute to the presence of sufficient rewards for engaging in replication. Among these structural conditions are the extent of the competition in the community, the potential for credit attribution and the authority of potential challengers to the new findings.

Kitcher's second example is that of choosing among two technical methods, where the community aim is the attainment of accurate results as soon as possible. In general, the community optimal is a division of individuals in which the use of both methods occurs, unless one of them is clearly superior. Individual decisions that give rise to the community optimal result depend on the extent to which the community members are motivated by epistemic versus personal goals. In the case of an epistemically pure community, individuals base their decision strictly on the probability that one method is intrinsically better, leading to the use of only that method. Contrast the community group composed of individuals seeking to maximize self-interest. Possibilities for reward may make it advantageous for individuals to try a less probable method in terms of intrinsic superiority. Although community consensus in favour of one method may be beneficial if the chosen method is clearly superior. Under conditions of uncertainty about the methods, a community optimal division of users is preferable so as not to prematurely eliminate a method that may contribute to the community aim of accurate results.

In this example, community structures (e.g. institutions) that provide incentives for the individual attainment of non-epistemic goals are more likely to promote the optimal diversity. Another possibility is the introduction of local autocratic figures to impose method choice. The result is a solution for maintaining diversity when consensus formation based on individual decisions is suboptimal for the community.

In concluding discussions, Kitcher summarized his theory by claiming first that non-epistemic motives contribute to the scientific community's epistemic goals by promoting diversity. Secondly, the effect of factors such as authority depends on the contextual variables including the social situation and the decision problem itself. The process of progressive change in consensus practice involves complex, individual reasoning efforts refined by the social situation. The combination of individual and social factors results in various distributions of cognitive effort. Understanding the effect of these distributions on community outcomes is essential for developing effective community strategies for advancing knowledge (Kitcher, 1993).

\section{Analysis, synthesis and preliminary model creation}

To summarize thus far, the selected theories were analysed for content and structure. Bayesianism provides an account of individual beliefs and their revision, given new evidence. Kitcher's (1993) theory of the OCL explains the relationship between individual beliefs, the role of authority and the formation of consensus practice in a community. The process of identifying concepts and statements about the relationships among them for each theory was iterative and often concurrent with synthesis, or combining the elements into a single model.

\section{Theory synthesis}

Following individual identification, the concepts from each theory were combined and then time ordered, 
Table I. Combined concepts from source theories.

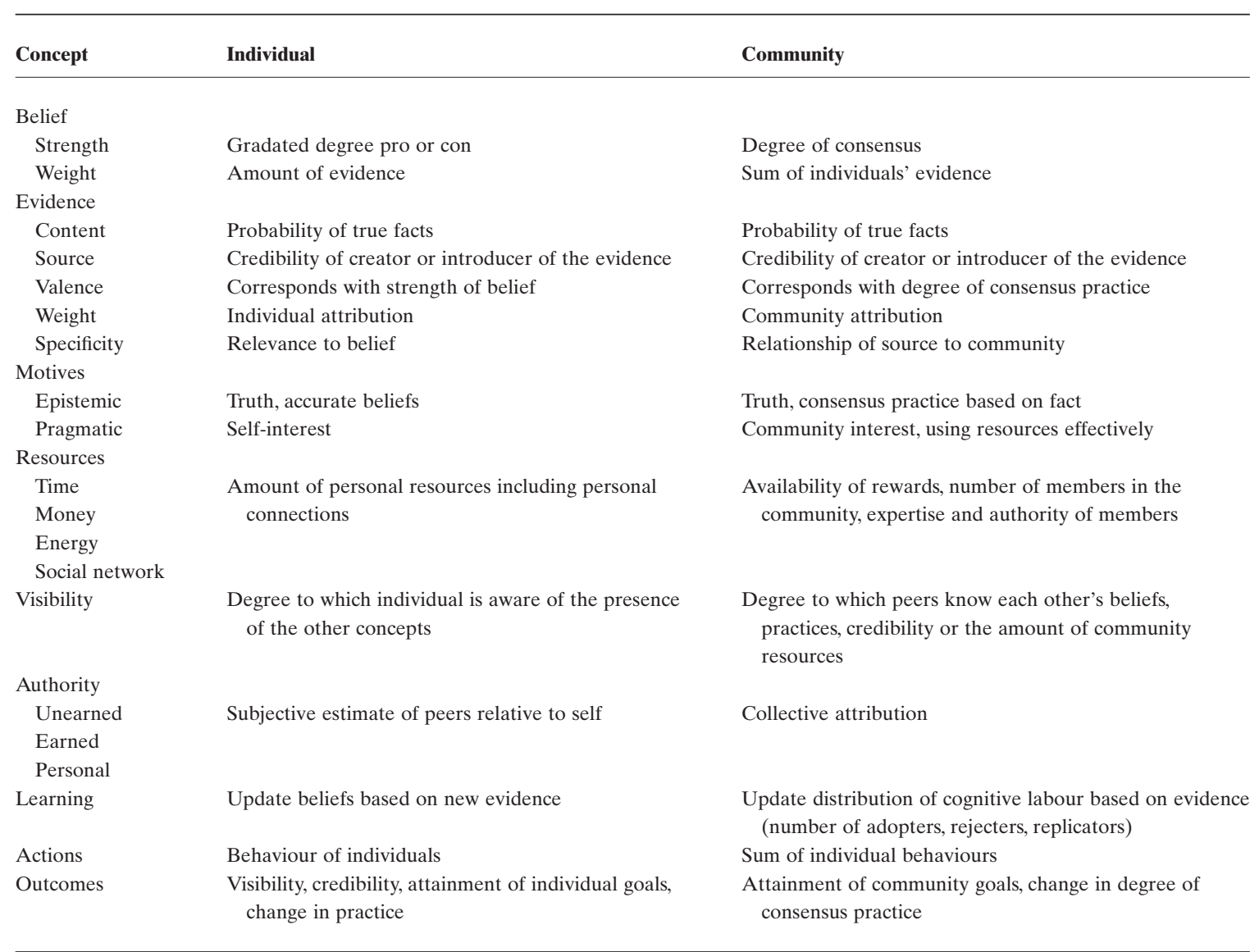

based on the statements that describe the relationship between the concepts. The provisional placement of the relational statements from each theory under the combined conceptual headings resulted in a master list of statements. Further refinement of the concept definitions resulted from the iterative process of working with the statements. For example, wording the relational statements in the form of 'if/then' relationships further aided identification and clarification of concepts derived from B \& OCL.

When defining concepts, it is important to recognize differences in terminology among and within theories. For example, the terms 'belief' and 'opinion' were used interchangeably and are considered synonymous here. The concept of 'evidence' encompasses actual evidence, new information, new practices and new findings. Pragmatic motives were also labelled 'practical', 'personal' and 'sullied'. Credit, or credibility, is the outcome of the attribution of authority and was used interchangeably to mean 'total authority'. Finally, the concept 'learning' is also known as 'conditioning', 'updating' or 'revising' beliefs. All of the nine concepts are variable and most are multidimensional. Table 1 presents the concepts, their major dimensions and examples of their multilevel meanings.

After initial clarification of the concepts and relational statements, the next step in the model development process was further analysis of the relational statements that were identified from the two theories. Each statement was categorized in terms of the nature of the relation as defined by Hardy (1973/2004). The majority were probabilistic (if A, then probably B). Many were also conditional (if A, then probably B, but 
only if C). Structural assessment of the concepts and relational statements revealed the presence of connections among all of the concepts, either directly or conditionally. The few relationships between the concepts that were not specifically derived from the analysis were theoretically deduced from the others (e.g. via time ordering). Comparison of the relational statements, removal of duplicates and reordering by sequence and level of abstraction resulted in nine concepts and 78 statements for the resulting synthesized theoretical model, the final phase of the content and structure selection step of TD.

\section{Description in nursing terms}

The final step in Walker \& Avant's (2005) TD procedure is the modification of the content and structures of the theory for use in nursing. Initial structural modification occurred via the synthesis of concepts and relational statements. The description of the resulting abstract model in nursing terms explicates its usefulness for the discipline. In order to achieve the aim of constructing the preliminary model suitable for use as a programming guide, the synthesized model is described as a narrative as well as being diagrammatically illustrated. The construction of the narrative provides a framework for the development of the agent-based model because it elucidates the attributes of various individuals and the processes used by them, within a given context. The diagrams were created using Unified Modeling Language (UML) in order to visualize the processes and logical connections in a time ordered fashion to aid programming of the dynamic agent-based model.

First, the following scenario illustrates the model in the context of a hospital setting (community). Within the hospital are several patient care units defined by a medical specialty (communities). Each unit has a manager, a clinical specialist and staff nurses with a range of nursing experience.

Nursing OL example:

To begin the process, Jane, the clinical specialist on the stroke unit, attends a hospital-wide meeting. At the meeting, Nancy, the clinical specialist from the orthopaedic unit, pre- sents information about a new nursing intervention to prevent pressure ulcers in immobilized patients (obtains new evidence). Initially, Jane is not really sure that the new intervention is much of an improvement over current practice (direct calibration of content), but her friend Nancy seemed enthusiastic and, it seems to Jane, usually knows what she is talking about (direct calibration of source, attribution of personal and earned authority) even though she is in a different specialty area (specificity). Jane is still uncertain about the new information; however, because of the renewed demands to reduce the occurrence of pressure ulcers resulting from new Medicare regulations (motives), and since her meeting with the manager was cancelled at the last minute, Jane decides to use the time (resources) to find out more about the intervention (investigate further).

Jane looks up the original research report and discovers that the study was a randomized controlled trial conducted at an eminent university by well-known researchers in the field (assess content and source of evidence). In light of what she found, Jane reassesses the evidence, determines that it is reliable and revises her opinion such that she is now strongly convinced that the new intervention is the best practice for preventing pressure sores. Given the potential benefit to immobilized patients on the unit and the kudos Jane will get for reducing the incidence of pressure ulcers on the unit, she requests time on the agenda at the next staff meeting (motives, action). At the meeting, Jane enthusiastically voices her opinion that she and everyone on the unit should adopt the new practice (visibility, change in consensus about practice).

At this point, the process of belief change for one individual is complete. The question of whether or not Jane becomes an opinion leader is dynamic and depends on multiple repetitions of the process among other nurses on the unit. Will Jane influence the rest of the staff to adopt the new intervention? How might she contribute to the decisions others make? What are some of the contextual factors that affect her influence?

First, by announcing her belief in the new intervention at the staff meeting, Jane's views became visible to the rest of the nursing staff and second, she introduced new information about the nursing intervention. Staff members on the unit will now begin the belief revision process by assessing this new information, based on what they already know (prior beliefs) about pressure ulcer prevention (content), and their 
assessment of Jane's credibility (source). Because Jane made her opinion known, nurses can compare their own beliefs with hers (direct calibration, earned authority). In addition, Jane's previous record of giving sound patient care advice (earned authority) and her position as a clinical specialist (unearned authority) contribute to her credibility among the staff. Some of the new graduate nurses had learned about the new intervention in school, although they were not very confident about it since no one on the unit used it (strength of belief, action)

This knowledge, combined with their belief in Jane as a credible source, led them to adopt the new information about the intervention and revise their own opinion. A number of the newcomers became convinced that the new intervention was a very good idea and visibly changed their own practice to incorporate the new intervention. This resulted in a change to the consensus practice on the unit. Others, although their opinions changed somewhat, were not quite convinced enough to put the new intervention into practice yet and kept their opinions to themselves.

Several of the more experienced nurses had a different reaction to the proposed new intervention. The intervention that was currently the consensus practice had worked perfectly fine over the years (strength and weight of belief). The new intervention did not seem likely to improve things that much (content) and furthermore, Jane had not actually taken care of a patient in months (source). In fact, they believed, it was nothing new at all, and so ignored the information.

Other nurses were less certain about the evidence for the new intervention. A number of them, although citing a lack of time (resources), thought that there was some potential for an improvement in patient care (motives). Rather than check the evidence themselves, they asked other nurses, believed to be credible, for their opinions about the new evidence. A few asked Jane about the credibility of her original sources (indirect calibration). Others decided to take the time to read the research report or to try out the intervention themselves before making up their minds. Armed with additional information about the evidence, the nurses reassessed it. Several adopted it and updated their own beliefs about nursing interventions to prevent pressure ulcers. Eventually, more nurses on the unit changed their practice to the new intervention, thereby revising community consensus practice.
The changing consensus on the unit pleased the manager, since even though it was taking some time, she was able to avoid imposing consensus by requiring the use of the new intervention (motives). Her public praise of Jane, for contributing to the reduction of pressure ulcers by introducing the new intervention, improved the clinical specialist's credibility among her peers. The fact that Jane acted on beliefs that turned out to be true was made even more visible by the accolades of the manager (earned authority). Soon clinical specialists from other units were asking Jane about the new intervention, expanding her influence (credible source of new evidence) and opening up new sources of information for herself (increasing resources).

The above example, although highly idealized, explains how the opinions and actions of individual nurses can affect the beliefs of others within the context of a patient care unit. To summarize the major points: nurses who are motivated to act on the strength of their opinions become a visible resource for their colleagues (beliefs, motives, visibility). The extent to which other nurses are aware of a co-worker's successful record of accomplishment in terms of acting on accurate beliefs contributes to their credibility (actions, outcomes, authority). If a nurse is known to be credible then her opinion is more likely to be sought by others who are engaged in the process of revising their beliefs (authority, learning). The emergence of an opinion leader is to some extent based on the perceived need for them and the presence of credible individuals to fill the role (resources). For example, if all of the nurses on a unit are confident in their own ability to assess new evidence, they may not seek another opinion. Alternatively, it is possible that the perception that no one on the unit is credible on the topic exists.

In parallel with the process of creating the narrative description of the synthesized theoretical model of OL, the UML diagrams were developed. Figure 1 shows the overall model, depicting belief revision within the context of a community. Figure 2 depicts the expanded version of the evidence assessment processes used in the overall model. It is important to note that the diagrams depict a single episode of exposure to new evidence and decision making by individuals about that evidence. It is only when this 


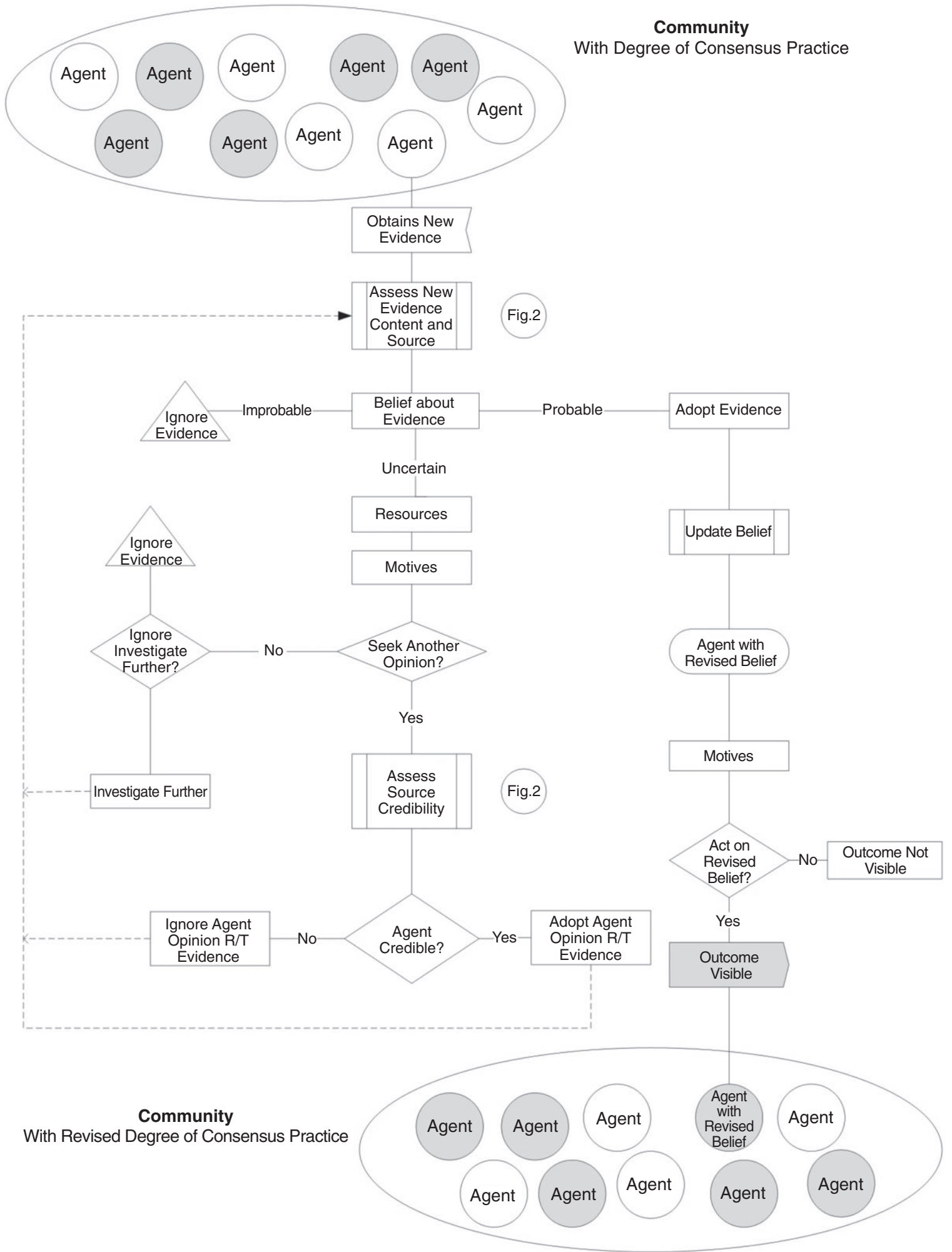

Fig. I. Synthesized model of individual and community belief revision 


\section{Assessing Evidence Content and Source Credibility}

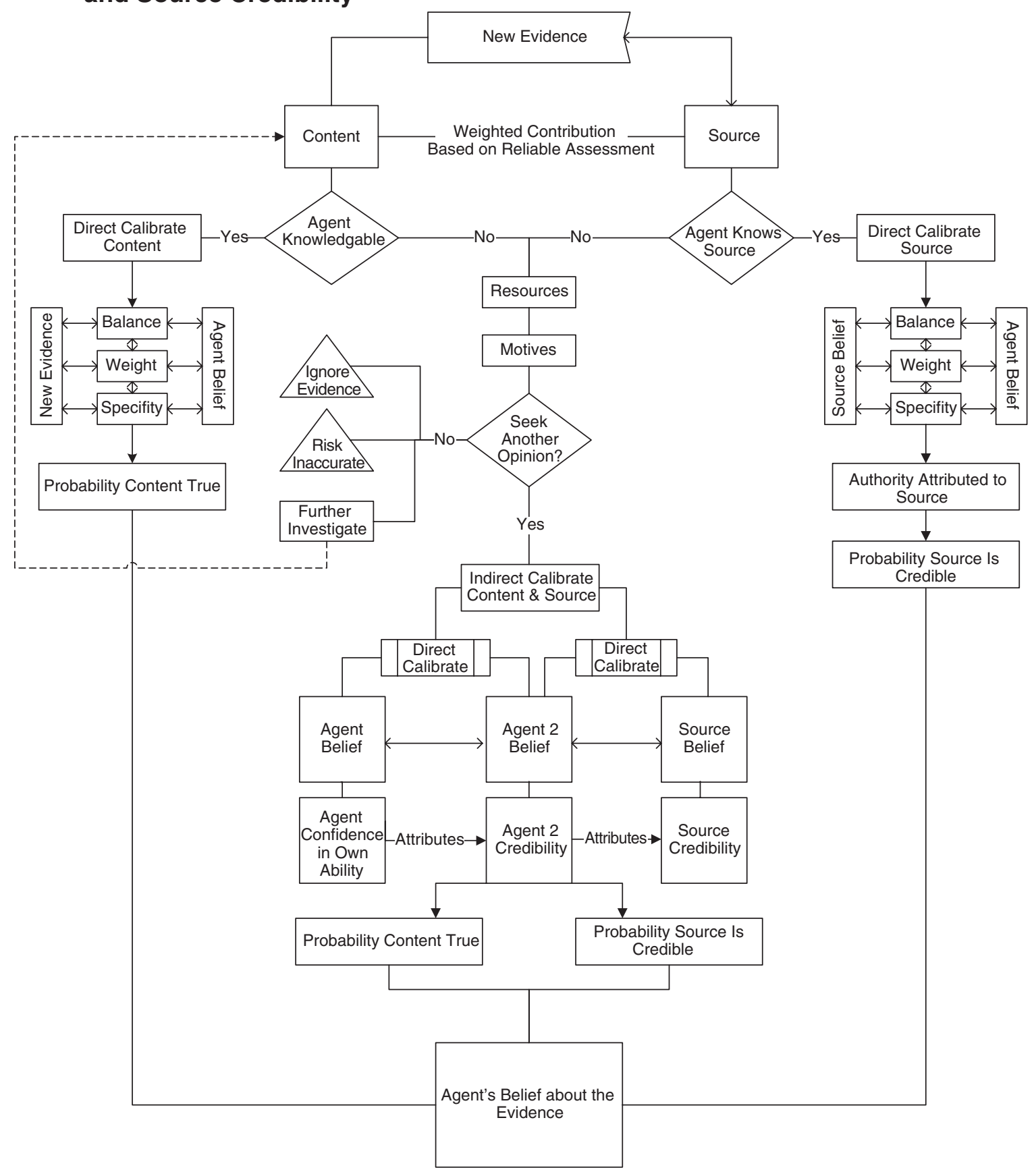

Fig. 2. Expanded processes represented in the overall model

process is repeated dynamically that an opinion leader (e.g. the person sought out for advice in Fig. 1) may emerge, depending on the characteristics of the other 'agents' in the context.
In summary, a combination of steps from Walker \& Avant's (2005) methods for theory construction formed the process used to develop a preliminary model of nursing OL in this paper. These steps 
included selection and analysis of philosophic theories for derivation, synthesis of the theories and construction of a representative model, further developed in nursing terms. The resulting model is abstract and contains concepts that are variable and multidimensional. In addition, the relationships among the concepts are probabilistic and often conditional on other probabilistic relationships. The ability to detect an opinion leader using the model as developed at this point is premature. According to Rogers's (2003) definition, OL is 'the degree to which an individual is able informally to influence other individuals' attitudes or overt behavior in a desired way with relative frequency' (p. 300). Using agent-based modelling as a tool for theory development allows the preliminary model to be viewed as a dynamic process. As a result the agent-based model explains how, and under what conditions, an individual who influences the opinions of others could emerge in a community over time. Additional insight about when and how influence on opinion formation works may contribute to strategies designed to increase an individual's influence and therefore his effect on the adoption of evidence-based practice changes in a community.

\section{Conclusions}

This model of nursing OL, developed by deriving a system of concepts and their relationships from philosophic theories, contributes to opinion leader theory by providing a normative framework for the development of an agent-based model designed to address gaps in understanding about the contextual factors on the development and utility of opinion leader strategies. In addition to enhancing explanation, the model development process highlighted several factors that make empirical testing difficult. These factors include the roles of context and multilevel interactions on the emergence of OL. In addition, the probabilistic, dynamic relationships among multidimensional and variable concepts pose challenges for research design as well. Simulated experiments using agent-based models provide an opportunity for gaining insight about complex processes such as those described here. For example, using an agent-based model it is possible to specify an individual's prior beliefs, the extent to which new evidence may contribute to revising those beliefs and how changing individual beliefs affects the community as a whole. In this paper, we described how theory construction techniques familiar to the nursing profession can be used to provide a foundation for developing theories using new methods such as agent-based modelling and simulation. Future research and testing of the model will help to determine the extent to which aspects of the model may need to be modified or further specified for use in nursing.

\section{References}

Anderson C.A. \& Whall A.L. (2011) A philosophical analysis of agent-based modelling: a new tool for theory development in nursing. Journal of Advanced Nursing, 67(4), 904-914.

Doumit G., Gattellari M., Grimshaw J. \& O'Brien M.A. (2007) Local opinion leaders. Cochrane effective practice and organisation of care group. Cochrane Database of Systematic Reviews, (1), CD000125.

Gilbert N. (2008) Agent-Based Models. Sage Publications, Thousand Oaks, CA.

Greenhalgh T., Robert G., Bate P., McFarlane F. \& Kyriakidou O. (2005) Diffusion of Innovations in Health Service Organisations: A Systematic Literature Review. Blackwell Publishing Ltd, Oxford.

Hardy M.E. (1973/2004). Theories: components, development, evaluation. In: Perspectives on Nursing Theory, 4th edn (eds P.G. Reed, N.B.C. Shearer \& L.H. Nicoll), pp. 75-94. Lippincott Williams and Wilkins, Philadelphia, PA.

Institute of Medicine Committee on Quality of Health Care in America (2001) Crossing the Quality Chasm: A New Health System for the 21st Century. National Academies Press, Washington, DC.

Joyce J.M. (1998) A nonpragmatic vindication of probabilism. Philosophy of Science, 65(4), 575-603.

Joyce J.M. (2004) Bayesianism. In: The Oxford Handbook of Rationality (eds A.R. Mele \& P. Rawling), pp. 132-155. Oxford University Press, New York.

Joyce J.M. (2005) How probabilities reflect evidence. Epistemology, Vol. 19, Philosophical Perspectives. Retrieved 20 February 2007, DOI: 10.1111/j.1520-8583.2005.00058.x

Kitcher P. (1993) The Advancement of Science: Science without Legend, Objectivity without Illusions. Oxford University Press, New York.

Miller J.H. \& Page S.E. (2007) Complex Adaptive Systems: An Introduction to Computational Models of Social Life. Princeton University Press, Princeton, NJ. 
Risjord M. (2009) Rethinking concept analysis. Journal of Advanced Nursing, 65(3), 684-691.

Rogers E.M. (2003) Diffusion of Innovations, 5th edn. Free Press, New York.

Walker L.O. \& Avant K.C. (1983) Strategies for Theory Construction in Nursing. Appleton-Century-Crofts, Norwalk, CT.

Walker L.O. \& Avant K.C. (1988) Strategies for Theory Construction in Nursing, 2nd edn. Appleton and Lange, Norwalk, CT.
Walker L.O. \& Avant K.C. (1995) Strategies for Theory Construction in Nursing, 3rd edn. Appleton and Lange, Norwalk, CT.

Walker L.O. \& Avant K.C. (2005) Strategies for Theory Construction in Nursing, 4th edn. Pearson-Prentice Hall, Upper Saddle River, NJ. 